\title{
RYMECse - An Android Application for Cse Department
}

\author{
Navyashree B $\mathbf{M}^{1}$, Soujanya $G^{2}$, Tanuja Patil ${ }^{3}$, Sai KrishnamRaju $G^{4}$, Sarvar Begum ${ }^{5}$ \\ Student, Computer Science, RYM Engineering College, Ballari, India ${ }^{1,2,3,4}$ \\ Assistant Professor, Computer Science, RYM Engineering College, Ballari, India ${ }^{5}$
}

\begin{abstract}
The application is designed for departmental use for our college where both the faculty members and students can register to seek the convenience to access digital library, digital notes, departmental events, faculty specialization and details, student details and registration forms linking to department events and many other add-ons. This application provides special features to faculty members to add notes in Digital notes section and add events commencing in department and links to register for these events. The users can access digital library, add any new books available in text field specified in digital library section, access co-faculty details in faculty specialization, add circulars in student section and can give their details specified with respect to semesters. Students are given permission only to register, login through University seat numbers and access all the sectional resources available exclusively for students.
\end{abstract}

Keywords: Role Based Key Access (RBAC), Authentication, Data Mining, MVC Architecture, Modules

\section{INTRODUCTION}

The main objective of this project is to add mobility and automation to the process of managing data in department. In a real world scenario, such as college campus, the information is in the form of notice, hand-written manual, verbal message and circulated among the student which is time consuming. So there is need to implement technology that promotes faster and easier communication among the students. The approach of communication is Android. The core idea of this project is to implement android based Mobile application for advancement of institution and educational system. The hybrid application will be used by students and faculty members. It includes features such as digital notes, digital library, event listings and faculty information. In the previous system, all the information has to view in a hard file, or in website and it doesn't include all the facilities that the proposed system provides. Hence, in order to overcome this problem, an Android Application is used to make this process easier, secure and search engine friendly.

\section{EXISTING SYSTEM}

The system which is used currently in the Rao Bahadur Y Mahabaleshwarappa Engineering College includes the manually submission of notes, announcing the commencing events through speech, borrowing books from library and viewing the faculty profile by approaching the particular faculty. Hence, the students and faculties approach of accomplishing the tasks involves much time and also results in lack of digitalization in the organization. The present system includes the college website where the ultimate need is to publicize the organization and includes college achievements and faculty profile which assigns permissions to the faculty only to update one's own profiles. In the organization current system, the notes are being shared with anyone of the student which in turn will be circulated to all the students. This process consumes more time. The announcement of commencing students is accomplished through manual speech and all the above mentioned tasks consumes more time and involves much of the physical works. Therefore, the solution for the proposed system is an android application where all the above tasks can be accomplished on the seats without manual work.

\section{DRAWBACKS OF EXISTING SYSTEM}

- In the existing system, there is no approach to download digital notes and download of digital book from library database.

- There is no option for the students to view the uploaded commencing events in the website.

- There is absence of users profile for viewing of the entire logged in user details.

- Absence of permissions to for faculties to upload notes in the website. 


\section{PROPOSED SYSTEM ARCHITECTURE}

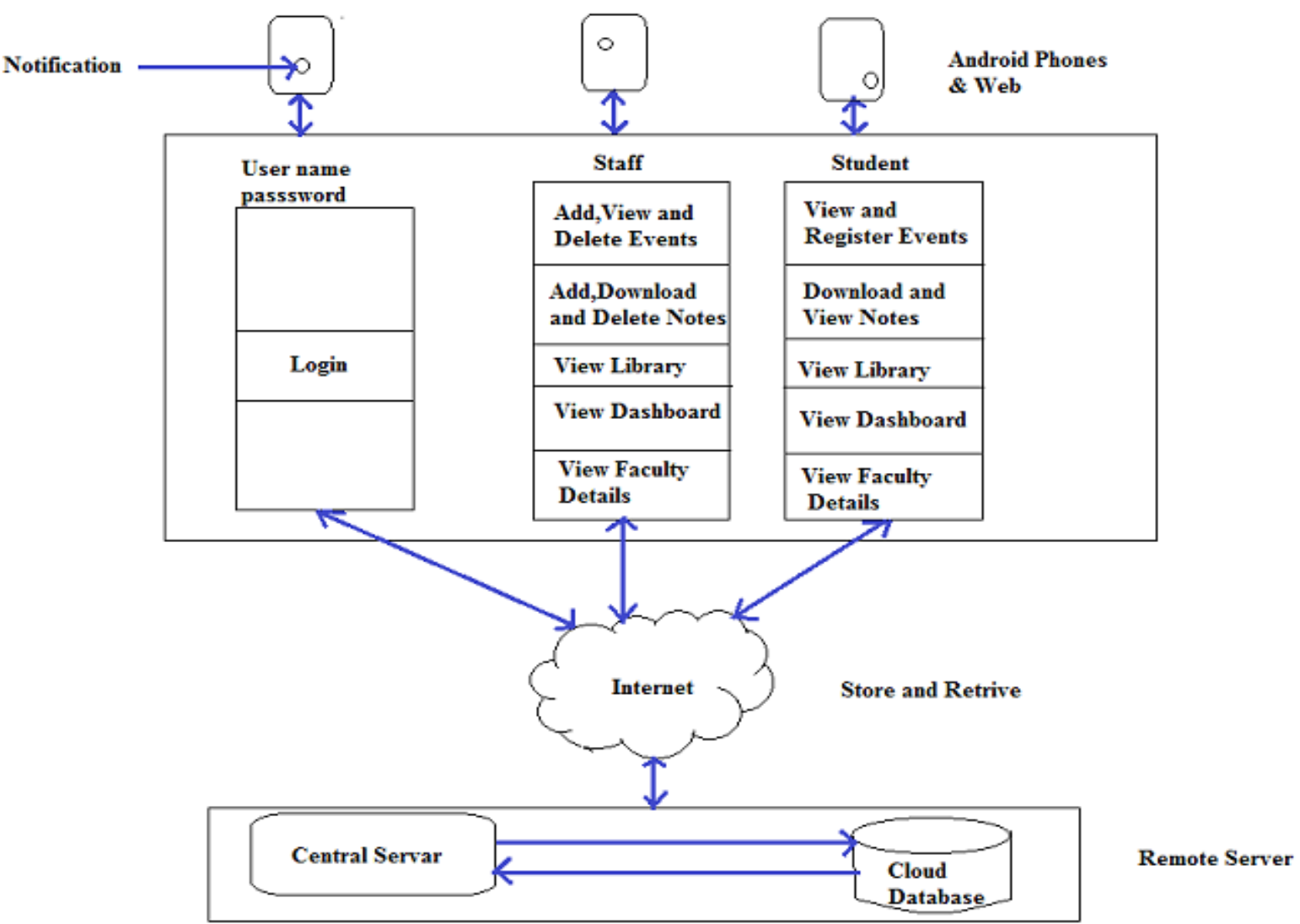

\section{Key Points}

The portal is strictly developed on MVC architecture principles.

The framework used to develop the portal allows us to have a single codebase and target multiple platforms.

The portal is deployment ready.

\section{METHODOLOGY}

\section{A. Cloud Database}

When a database server is running in machine using private IP (10.0.0.0 to $10.255 .255 .255,172.16 .0 .0$ to 172.31.255.255, 192.168.0.0 to 192.168.255.255, 127.0.0.1-localhost) the database is accessible only within that network. i.e. the database cannot be used by general public. The portal is hosted on public cloud on a public IP, thus anyone with internet connection with required privileges can read/write the database. The cloud database is nonrelational (no-sql) based DB, thus it doesn't have a schema and gives users' flexibility in data formats. The cloud database not only hosts data (ASCII text), it also hosts files upon a separate file system. Thus, users can upload, download and delete to this file system on the fly. All file formats are allowed. The cloud is tuned to provide high availability and low latency. The cloud database also handles asynchronous read/write call from multiple users from website or app which helps the database to be corrupt free and consistent across all platforms

(Website, android, etc.)

\section{B. Role Based Access Control (RBAC)}

The application will be dedicated for two sets of users

- Faculties

\section{- Students}

For faculty community to register a pre-shared key shared among the faculty community has to be used, thus barring others (students) from registering to portal as faculty. The faculties can login using the email and password set during the registration. The student community can register and login to the portal using their USN and password of their choice. Each set of user has to be given certain privileges on the cloud database. The RBAC feature helps in setting granular control over the entire application across platforms. This feature also keeps track of each user's last transaction from the application. 
Vol. 8, Issue 5, May 2019

\section{Search Engine for Books}

The portal provides an interface through which the student and faculty community can search for Books across the Internet. The engine will fetch the web and search for that particular query. The result includes all the metadata (name, author, is bns, publication, pages, language etc.) of books and also provides an interface for users to read them. The query can range from computer science to politics and novels. The engine will return books relevant to search query. The engine can be customized to search based on relevance and number of results.

\section{Data mining (faculty data)}

The portal will mine RYMEC's official web page to get all the information about faculties. The mined data will undergo filtering and will be formatted for consumption of the application. The formatted information (name, qualification, work experience, technical papers, and technical groups) will then be stored on cloud database for public access. The mining operation is performed using Python 3 script in the cloud. The portal also provides an intuitive and reactive search bar so that the users can easily search the faculties by name

\section{MODULES}

\section{A. User module}

The user modules are of student and faculty, the users are authenticated with usn and password in student section and the faculty is authenticated with Gmail and password. To make a difference between faculty and student the faculty is provided with a key at the sign-up process .if the provided authentication details are valid the user will be taken to their dashboard

\section{B. Database module}

The proposed system uses cloud-database module because of their storage and scalability. This module store information of library section, college event section, faculty details, and model their data on specified operations. These specific operations can store the data into the cloud.

\section{Event module}

In this section the user can come to know all the events that are held in the department, the events are added to event list by the faculty with the date, time along with its location, and permissions are given to faculty to delete from the event list by just sliding the event to left side. In the event list along with the list of events the user can also get the date on which the event is held and the numbers of days for the event to take place, along with the venue where the event is held. The event can be viewed by the user without any refreshment.

\section{Notes module}

With respect to this section the faculty has a permission to upload the notes, which can be accessed by the user. In this section the faculty will post the notes with respect to semester wise, with the priority levels. Get the live notes updates without refresh, this section allows all the file formats. The user can search the notes by name from the notes listing. The faculty can add notes to notes list with from validation, the delete notes from the notes listing can only be done by faculty.

\section{E. Library module}

With the help of search engine the user can find the required textbooks form the library module, which is stored in the cloud .In this application the list of books from any category with search filters can be used by the user to get the required textbook. Metadata of books that is the user can find the required textbook by the name of the textbook are by the name of the authors, publishers etc.

\section{F. Faculty module}

This section provides the user about the faculty details which includes qualification, work experience, the details of the technical papers published. The user can get the faculty details by searching the faculty by name, the faculty data are mined from official RYMEC website.

\section{WORK FLOW}

The detailed workflow of this application is as follows: The application will be dedicated for two sets of users-

A. Faculty/ Teaching Staff

1].Faculty Registration:

The first step in this application is Registration (Sign Up), Staff Members/Teaching faculty has to register through Preshared key provided to faculty community. The respective person will then provide his or her E-mail id and password for registration along with Key provided. 
Vol. 8, Issue 5, May 2019

\section{2].Faculty Login:}

After registering the faculty is allowed to $\log$ in. He or she can now view home Page (Dashboard) where there are options to Publish, delete and download notes. They can Publish, delete and view Events. View and download books in Library section and can view details of Faculty members in Faculty section.

\section{3]. View Dashboard:}

It is the home page for logged in users. Shows the metadata (login, role, and using portal duration) of the logged user and User guides for each section in the portal.

\section{4]. Faculty-Notes:}

The faculty community is given privilege to publish and delete notes. The published notes metadata will be stored in cloud database and the file in cloud file system. The metadata for each notes include name of publisher, file name, semester, subject, priority and upload time. Once the events are published, the listing is updated automatically without refresh across all users and all platforms instantaneously. This metadata will be the input to portal and has to be given using the reactive notes form. The community can filter through the notes using filter feature which filters notes by semester. The RBAC ensures that only faculties can publish and delete events, the student community is notified about events. The portal also provides an intuitive and reactive search bar so that the users can easily search the events by name.

\section{5].Faculty-Event:}

The faculty community is given privilege to publish and delete events related to college. The published event will be stored in cloud database. The metadata for each event includes event name, description, host name, location and start date. This metadata will be the input to portal and has to be given using the reactive events form. Once the events are published, the event listing is updated automatically without refresh across all users and all platforms instantaneously. The RBAC ensures that only faculties can publish and delete events, the student community is notified about events. The portal also provides an intuitive and Reactive search bar so that the users can easily search the events by name.

\section{6]. Faculty Details:}

The portal will mine RYMEC's official webpage to get all the information about faculties. The mined data will undergo filtering and will be formatted for consumption of the application. The formatted information (name, qualification, work experience, technical papers, and technical groups) will then be stored on cloud database for public access. The portal also provides an Intuitive and reactive search bar so that the users can easily search the faculties by name.

\section{B. Student}

\section{1]. Student Registration:}

In this initial step, the student has to complete the sign-up to avail the benefits of the application. The student has to register through their respective USN and Password. Here the student with USN $3 \mathrm{vc}^{* * * * * * *}$ only is made to signup, as the application is solely made for our college students and $3 \mathrm{vc}$ represents college code by university.

\section{2]. Student Login:}

After registering the Student is allowed to log in. The candidate can now view home Page (Dashboard) where there are options to download notes, view and register for Events. View and download books in Library section and can view details of Faculty members.

\section{3].View Dashboard:}

It is the home page for logged in users. It shows the metadata (login, role, and using portal duration) of the logged user and User guides for each section in the portal. This is same for both the users i.e. Faculty and Students.

\section{4].Student-Notes:}

The faculty community is given privilege to publish and delete notes. The metadata for each notes include name of publisher, file name, semester, subject, priority and upload time. Once the events are published, the listing is updated automatically without refresh across all users and all platforms instantaneously. The portal provides complete control over file upload, thus we can pause an upload and resume it later. This metadata will be the input to portal and has to be given using the reactive notes form. The community can filter through the notes using filter feature which filters notes by semester. The portal also provides an intuitive and reactive search bar so that the users can easily search the events by name. 
Vol. 8, Issue 5, May 2019

5]. Student-Event:

Student community can only view and register for events.

6]. Faculty Details:

Students can view - faculty details which are mined from RYMEC official website.

\section{BENEFITS OF PROPOSED SYSTEM}

- The Proposed System is digitalized and time saving it allows the students and Faculty to interact with respect to Department issues and updates, which will be easy and convenient to use.

- In faculty Login there is a key provided, which gives security for faculty login.

- Only the faculty has the permissions to update and delete in events and notes section.

- In notes section faculty can add notes with priority in semester vise.

- In library section we have search bar where the student can search for the required book and even displays related text books with download option.

- In events section we have description of event, host, location and it also shows the countdown of days with respect to event.

\section{CONCLUSION}

Application is Cloud based (web + app) and Data driven. Includes Reactive forms - Reactive to user inputs. Has Cloud storage (text) and database (files). Has RBAC inculcated. there is a Live status with Granular control for file upload and Sockets and Threads running Background sync with db without refresh or reopen which facilitates user to have more friendly environment to work. Application saves Individual's Time, provides Authorized Access to Faculty as well as Students. Cloud thus accomplishes friendly search engine.

\section{REFERENCES}

[1]. Rymec.in- official site of RYMEC.

[2]. RYMEC -official Android application of RYMEC.

[3]. csedatahub.blogspot.com- official site for CSE department faculty members of RYMEC.

[4]. "dhi"- An android application for Adhichunchanagiri institute of technology.

[5]. A.J.Kadam, "Mobile Web Based Android App for College Management system," in IEEE IJECS Vol.6 Iss.2 Feb,2017, pp. $20206-20209$. 